\title{
Unitary pole approximation for the Coulomb-plus-Yamaguchi potential and application to a three-body bound-state calculation
}

\author{
K. Ueta \\ Instituto de Física da Universidade de São Paulo, Caixa Postal 20516, 01498 São Paulo, Brazil \\ G. W. Bund \\ Instituto de Física Teórica, Universidade Estadual Paulista, Rua Pamplona, 145, 01405 São Paulo, Brasil
}

(Received 28 December 1990)

\begin{abstract}
The unitary pole approximation is used to construct a separable representation for a potential $U$ which consists of a Coulomb repulsion plus an attractive potential of the Yamaguchi type. The exact bound-state wave function is employed. $U$ is chosen as the potential which binds the proton in the $1 d_{5 / 2}$ single-particle orbit in ${ }^{17} \mathrm{~F}$. Using the separable representation derived for $U$, and assuming a separable Yamaguchi potential to describe the $1 d_{5 / 2}$ neutron in ${ }^{17} \mathrm{O}$, the energies and wave functions of the ground state $\left(1^{+}\right)$and the lowest $0^{+}$state of ${ }^{18} \mathrm{~F}$ are calculated in the core-plustwo-nucleons model solving the Faddeev equations.
\end{abstract}

\section{INTRODUCTION}

In some three-body processes, the $T$ matrix associated to a pair of particles is dominated by the bound-state pole of the pair. In this case the interaction $U$ between these particles can be approximated by the separable potential

$$
U_{\text {sep }}=U\left|\Psi^{\mathrm{BS}}\right\rangle\left\langle\Psi^{\mathrm{BS}}|U| \Psi^{\mathrm{BS}}\right\rangle^{-1}\left\langle\Psi^{\mathrm{BS}}\right| U,
$$

$\left|\Psi^{\mathrm{BS}}\right\rangle$ being the bound-state wave function. This approximation is known as unitary pole approximation (UPA).

Our purpose is to construct, based on the UPA, a separable approximation for a potential $U$ which consists of a short-range attractive part $V$ and of a Coulomb repulsion $V_{C}=\left(Z Z^{\prime} e^{2}\right) / r$. As is well known, the inclusion of the Coulomb interaction in the three-body problem presents many difficulties. Although it has been possible to extend the Faddeev formalism to include the Coulomb force, ${ }^{1}$ the numerical applications have been restricted to low values $(\lesssim 4)$ of the product $Z Z^{\prime} .^{2,3}$ The usual replacement of the Coulomb $T$ matrix by $V_{C}$ (Born approximation) becomes questionable as $Z Z^{\prime}$ increases.

In a recent calculation of the $p$ - $d$ breakup reaction, ${ }^{4}$ the long-range tail of the Coulomb interaction is replaced by a short-range potential and the Ernst-Shakin-Thaler (EST) method $^{5}$ is used to obtain a separable approximation for this cutoff Coulomb potential. The EST method is more general than the UPA. However, for the UPA it is not necessary to make any screening of the Coulomb tail.

\section{CONSTRUCTION OF THE UPA FORM FACTOR}

Having in mind applications to three-body systems consisting of two nucleons outside an inert and massive core, we consider $U$ as being the single-particle potential of the proton. For simplicity, it will be assumed that the short-range part $V$ is already separable and acts in a specific $(l j)$ orbit of the shell model:

$$
\left\langle\mathbf{p}|V| \mathbf{p}^{\prime}\right\rangle=-\frac{\lambda_{l j}}{2 m} g_{l j}(p) g_{l j}\left(p^{\prime}\right) \sum_{\mu}\left\langle\hat{\mathbf{p}} \mid \mathcal{Y}_{l j \mu}\right\rangle\left\langle\mathcal{Y}_{l j \mu} \mid \hat{\mathbf{p}}^{\prime}\right\rangle,
$$

where $\mathbf{p}$ is the momentum of the proton (mass $m$ ) and

$$
\left\langle\hat{\mathbf{p}} \mid \mathcal{Y}_{l j \mu}\right\rangle=\sum_{m_{l} m_{s}}\left(\operatorname{lm}_{l} \frac{1}{2} m_{s} \mid j \mu\right) Y_{l}^{m_{l}}(\hat{\mathbf{p}})\left|\frac{1}{2}, m_{s}\right\rangle .
$$

We assume also that the error made in considering the core as a point charge, which leads to an excess Coulomb repulsion in the region corresponding to the interior of the core, is compensated for by making the potential $V$ more attractive.

It is convenient to choose a form factor of the Yamaguchi type,

$$
g_{l j}(p)=\frac{p^{l}}{\left(p^{2}+\beta^{2}\right)^{l+1}} .
$$

With this choice, the two-body problem corresponding to the potential $V+V_{C}$ can be solved exactly. ${ }^{6,7}$ The energy $\epsilon_{l j}$ of the bound state is determined by the equation

$$
\begin{aligned}
\lambda_{l j}^{-1}= & \frac{\pi}{2} 4^{-l}\left(\begin{array}{c}
2 l+1 \\
l
\end{array}\right) \frac{l+1}{l+1-s / \kappa}(2 \beta)^{-2 l-1}(\beta+\kappa)^{-2} \\
& \times_{2} F_{1}\left[1,-l-\frac{s}{\kappa} ; l+2-\frac{s}{\kappa} ;\left(\frac{\beta-\kappa}{\beta+\kappa}\right)^{2}\right),
\end{aligned}
$$

where $s=-\left(2 m Z Z^{\prime} e^{2}\right) / 2$ and $\kappa=\left(2 m\left|\epsilon_{l j}\right|\right)^{1 / 2}$. The corresponding wave function is given by

$$
\Psi_{l j \mu}^{\mathrm{BS}}(\mathbf{p})=N_{l j} \frac{1}{p^{2}+\kappa^{2}}\left[g_{l j}(p)-v_{l j}^{C}(p)\right] \mathcal{Y}_{l j \mu}(\widehat{\mathbf{p}}),
$$

where 


$$
\begin{aligned}
v_{l j}^{C}(p)=- & \frac{1}{p}\left[\frac{4 p \kappa^{2}}{\left(p^{2}+\kappa^{2}\right)\left(\beta^{2}-\kappa^{2}\right)}\right]^{l+1}, \\
& \times \sum_{n=l+1}^{\infty} \frac{s / \kappa}{n-s / \kappa}\left[\frac{\beta-\kappa}{\beta+\kappa}\right]^{n} C_{n-l-1}^{l+1}\left(\frac{p^{2}-\kappa^{2}}{p^{2}+\kappa^{2}}\right),
\end{aligned}
$$

and $N_{l j}$ is a normalization factor. The $C_{n}^{m}(z)$ are the Gegenbauer polynomials.

From expression (6) we see that the separable potential $U_{\text {sep }}$, which generates the same bound state as the potential $V+V_{C}$, is given by

$$
\left\langle\mathbf{p}\left|U_{\text {sep }}\right| \mathbf{p}^{\prime}\right\rangle=-\frac{\Lambda_{l j}}{2 m} g_{l j}^{C}(p) g_{l j}^{C}\left(p^{\prime}\right) \sum_{\mu}\left\langle\hat{\mathbf{p}} \mid \mathscr{Y}_{l j \mu}\right\rangle\left\langle\mathcal{Y}_{l j \mu} \mid \hat{\mathbf{p}}^{\prime}\right\rangle,
$$

with

$$
g_{l j}^{C}(p)=g_{l j}(p)-v_{l j}^{C}(p),
$$

and $\Lambda_{l j}$ is determined by requiring that the bound state has the energy eigenvalue $\epsilon_{l j}$. Expression (8) is the UPA [Eq. (1)] when the degeneracy introduced by the quantum number $\mu$ is included.

As an example, we consider $U$ as the interaction which describes the $1 d_{5 / 2}$ single-particle bound state of the proton in ${ }^{17} \mathrm{~F}$. The energy of the bound state $\left(\epsilon_{25 / 2}=-0.596 \mathrm{MeV}\right)$ is reproduced if we take $\lambda_{25 / 2}=945 \mathrm{fm}^{-7}$ and $\beta=1.464 \mathrm{fm}^{-1}$ in Eqs. (2) and (4). The value of $\beta$ is the same as the one appropriate for the neutron $1 d_{5 / 2}$ single-particle state in ${ }^{17} \mathrm{O}$ (see Sec. III). In Fig. 1 we show the radial function

$$
\begin{gathered}
u(r)=A r^{3} / e^{-\kappa r} \int_{0}^{1} x^{2+i \gamma}\left(1+\frac{\beta-\kappa}{2 \kappa} x\right)^{2-i \gamma} \\
\times e^{-(\beta-\kappa) r x} d x,
\end{gathered}
$$

where $A$ is a normalization constant and $\gamma=i s / \kappa$ (Ref. 7, p. 432). The corresponding mean-square radius is 3.74 $\mathrm{fm}$. This value is very close to the value $3.69 \mathrm{fm}$ given in Ref. 8. In Fig. 2 we plot the functions $g_{25 / 2}(p), v_{25 / 2}^{C}(p)$, and $g_{25 / 2}^{C}(p)$ [Eqs. (4), (7), and (9)]. It can be shown analytically that $v_{25 / 2}^{C}(p)$ behaves as $p^{2}$ when $p \rightarrow 0$ and as $p^{-4}$ for $p \rightarrow \infty$. Finally, we mention that the coupling

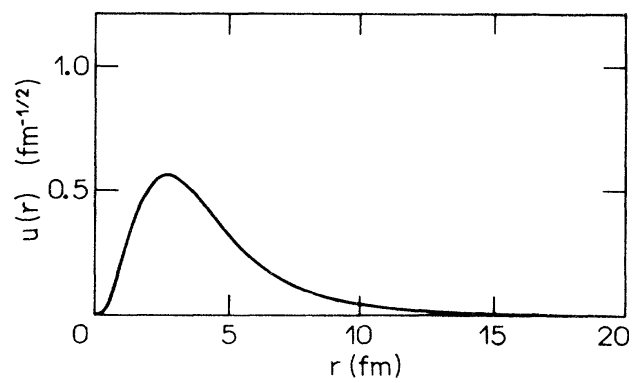

FIG. 1. Exact radial wave function for the $1 d_{5 / 2}$ state in the Coulomb-plus-Yamaguchi potential.

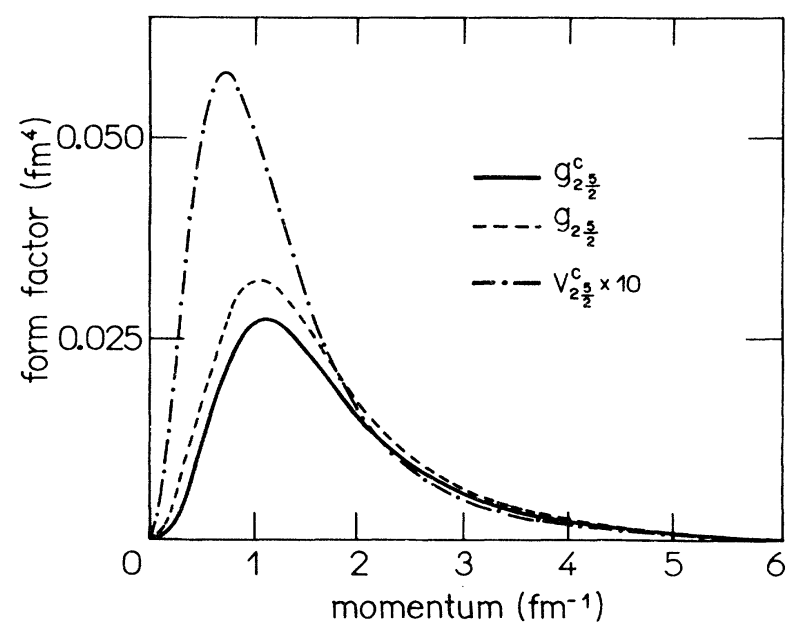

FIG. 2. Form factors $g_{25 / 2}^{C}$ of UPA, $g_{25 / 2}$ of the Yamaguchi potential, and the difference $v_{2}^{C}{ }_{5 / 2}$ between $g_{25 / 2}$ and $g_{25 / 2}^{C}$.

constant $\Lambda_{25 / 2}$ which appears in Eq. (8) results in being equal to $1103 \mathrm{fm}^{-7}$.

\section{APPLICATION \\ TO A THREE-BODY BOUND-STATE CALCULATION}

We consider the nucleus ${ }^{18} \mathrm{~F}$ as a three-body system composed of an ${ }^{16} \mathrm{O}$ core plus a proton (particle 1) and a neutron (particle 2). We restrict ourselves to bound states dominated by the $\left(1 d_{5 / 2}, 1 d_{5 / 2}\right)$ configuration and, in fact, consider only the ground state $\left(1^{+}\right)$and the lowest $0^{+}$state (excitation energy $1.042 \mathrm{MeV}$ ).

For the proton- ${ }^{16} \mathrm{O}$ interaction $U_{1}=V_{1}+V_{C}$, we use the UPA potential described in the previous section. For the neutron-core interaction $V_{2}$, a potential of the same form as the short-range part $V_{1}$ of the proton interaction [Eqs. (2)-(4)] is used. The parameters are chosen in such a way to reproduce the energy $(-4.146 \mathrm{MeV})$ and the radius ${ }^{9}(3.464 \mathrm{fm})$ of the $1 d_{5 / 2}$ single-particle state in ${ }^{17} \mathrm{O}$. Thus the values $\lambda_{25 / 2}^{(2)}=924 \mathrm{fm}^{-7}$ and $\beta_{25 / 2}^{(2)}=1.464 \mathrm{fm}^{-1}$ are obtained. We here make the remark that since $\lambda_{25 / 2}^{(1)}$ $\left(=945 \mathrm{fm}^{-7}\right)$ is larger than $\lambda_{25 / 2}^{(2)}, V_{1}$ is more attractive than $V_{2}$. This is expected since, as was pointed out in Sec. II, $V_{1}$ has an additional attractive part to compensate for the excess Coulomb repulsion.

For the neutron-proton interaction $V_{12}$, we use the separable $s$-wave Yamaguchi potential

$$
\begin{aligned}
& \left\langle\mathbf{q}\left|V_{12}\right| \mathbf{q}\right\rangle=-\sum_{S=0,1} \frac{\Lambda_{S}}{m} g_{S}(q) g_{S}\left(q^{\prime}\right) \\
& \times \sum_{M_{S}}\left|S M_{S}\right\rangle\left\langle S M_{S}\right| \\
& g_{S}(q)=\frac{1}{q^{2}+\beta_{S}^{2}} .
\end{aligned}
$$

In Eq. (11), $\mathbf{q}$ is the relative momentum $\frac{1}{2}\left(\mathbf{P}_{1}-\mathbf{P}_{2}\right), \mathbf{P}_{i}$ being the momentum of particle $i$, and $\left|S M_{S}\right\rangle$ is the spin 


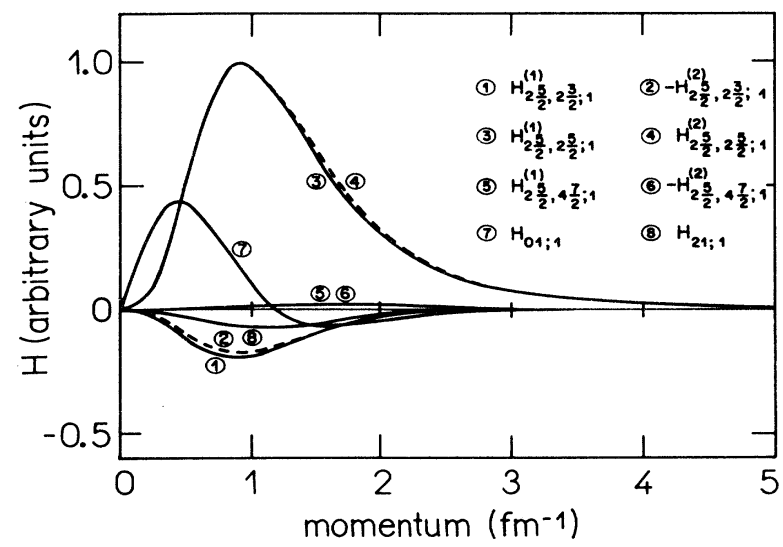

FIG. 3. Spectator functions for the ground state of ${ }^{18} \mathrm{~F}$.

wave function for the proton-neutron system. The values used for the parameters are $\Lambda_{0}=0.149 \mathrm{fm}^{-3}, \beta_{0}=1.165$ $\mathrm{fm}^{-1}, \Lambda_{1}=0.382 \mathrm{fm}^{-3}$, and $\beta_{1}=1.406 \mathrm{fm}^{-1}$, which are determined from the values $a_{s}=-23.71 \mathrm{fm}, r_{0 s}=2.70$ $\mathrm{fm}, a_{t}=5.42 \mathrm{fm}$, and $r_{0 t}=1.76 \mathrm{fm}$ for the scattering

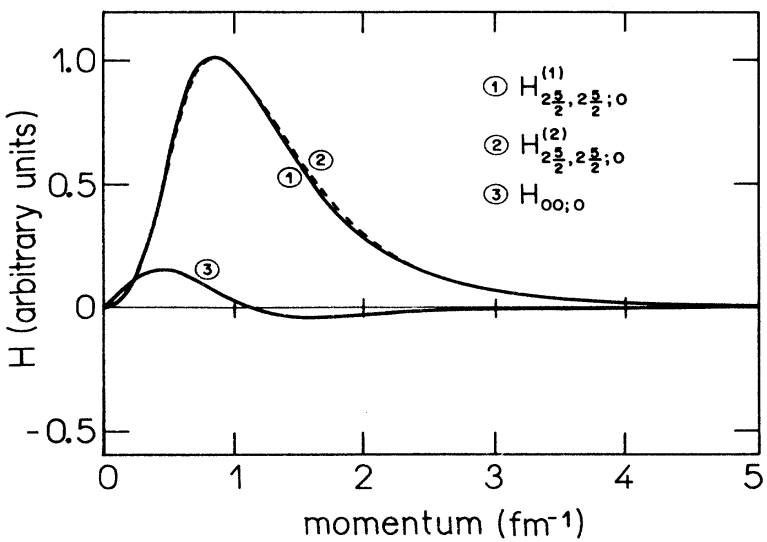

FIG. 4. Spectator functions for the lowest $0^{+}$state of ${ }^{18} \mathrm{~F}$.

length and effective range of the neutron-proton scattering.

Performing the Faddeev decomposition of the total wave function, $\Psi=\Psi^{(1)}+\Psi^{(2)}+\Psi^{(3)}$, we obtain the following expressions for the components $\Psi^{(i)}$ :

$$
\begin{aligned}
& \Psi^{(1)}\left(\mathbf{P}_{1}, \mathbf{P}_{2}\right)=\frac{2 m}{2 m E-P_{1}^{2}-P_{2}^{2}} \sum_{l^{\prime} j^{\prime}} g_{2}^{C}{ }_{5 / 2}\left(P_{1}\right) \frac{H_{25 / 2, l^{\prime} j^{\prime} ; J}^{(1)}\left(P_{2}\right)}{P_{2}} \mathscr{Y}_{25 / 2, l^{\prime} j^{\prime} ; J M_{J}}\left(\widehat{\mathbf{P}}_{1}, \widehat{\mathbf{P}}_{2}\right), \\
& \Psi^{(2)}\left(\mathbf{P}_{1}, \mathbf{P}_{2}\right)=\frac{2 m}{2 m E-P_{1}^{2}-P_{2}^{2}} \sum_{l^{\prime} j^{\prime}} g_{25 / 2}^{(2)}\left(P_{2}\right) \frac{H_{25 / 2, l^{\prime} j^{\prime} ; J}^{(2)}\left(P_{1}\right)}{P_{1}} \mathscr{Y}_{l^{\prime} j^{\prime}, 2}{ }_{5 / 2 ; J M_{J}}\left(\widehat{\mathbf{P}}_{1}, \widehat{\mathbf{P}}_{2}\right), \\
& \Psi^{(3)}(\mathbf{P}, \mathbf{p})=\frac{2 m}{2 m E-\frac{1}{2} P^{2}-2 p^{2}} \sum_{L S} \sqrt{4 \pi} g_{S}(p) \frac{H_{L S ; J}(P)}{P} Y_{L O(L) S ; J M_{J}}(\widehat{\mathbf{P}}, \hat{\mathbf{p}}) .
\end{aligned}
$$

In Eqs. (13)-(15), $E$ is the energy of the three-body bound state, $\left(J, M_{J}\right)$ denotes the total angular momentum, $\mathbf{P}$ is the center-of-mass momentum $\mathbf{P}_{1}+\mathbf{P}_{2}$, and the $\mathcal{Y}$ 's are the usual total angular momentum eigenfunctions.

The spectator functions $H$ satisfy the homogeneous integral equations given in Refs. 10 and 11 . For the $1^{+}$ state, we have $\left(l^{\prime}, j^{\prime}\right)=\left(2, \frac{5}{2}\right),\left(2, \frac{3}{2}\right),\left(4, \frac{7}{2}\right), L=0,2$, and $S=1$ in expansions (13)-(15) and, for the $0^{+}$state, $\left(l^{\prime}, j^{\prime}\right)=\left(2, \frac{5}{2}\right), L=0$, and $S=0$. The coupled integral equations are transformed into a system of algebraic equations by applying the $N$-point Gauss quadrature method for the integrals. The vanishing of the associated determinant gives the energy eigenvalue. In this way we get $E_{1^{+}}=-10.31 \mathrm{MeV}$ and $E_{0^{+}}=-7.93 \mathrm{MeV}$. These numbers are close to the experimental values ${ }^{12}$ $E_{1^{+}}^{\text {expt }}=-9.75 \mathrm{MeV}$ and $E_{0^{+}}^{\text {ext }}=-8.71 \mathrm{MeV}$, despite the fact that only the $1 d_{5 / 2}$ interaction is considered.

In order to evaluate the contribution of the Coulomb force to the three-body bound-state energy, we replace the valence proton by a neutron and calculate the energy of the $0^{+}$ground state of ${ }^{18} \mathrm{O}$ obtaining $E_{0^{+}}$ $\left({ }^{18} \mathrm{O}\right)=-11.38 \mathrm{MeV}$. Therefore, in our model, the switching on of the proton-core Coulomb interaction raises the energy by an amount $E_{0^{+}}\left({ }^{18} \mathrm{~F}\right)$ $-E_{0^{+}}\left({ }^{18} \mathrm{O}\right)=-7.93 \mathrm{MeV}+11.38 \mathrm{MeV}=3.45 \mathrm{MeV}$. Ex-

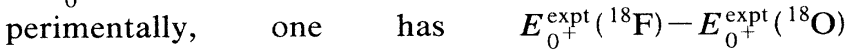
$=-8.71 \mathrm{MeV}+12.19 \mathrm{MeV}=3.48 \mathrm{MeV}$. This shows that the UPA is able to yield a correct value of the Coulomb energy.

In Figs. 3 and 4, we plot the spectator functions versus momentum. From the closeness of $H^{(1)}$ and $H^{(2)}$, we conclude that the asymmetry introduced in the total wave function [Eqs. (13)-(15)] by the Coulomb force lies mainly in the difference $v_{25 / 2}^{C}$ between the form factors $g_{25 / 2}^{C}$ and $g_{25 / 2}^{(2)}\left(g_{25 / 2}\right.$ in Fig. 2) and is about 20\%. We make here the remark that in the numerical calculations we found it convenient to multiply $g_{25 / 2}^{C}$ and $g_{25 / 2}^{(2)}$ by a factor of 15.50 and, accordingly, divide $\Lambda_{25 / 2}$ and $\lambda_{25 / 2}^{(2)}$ by $(15.50)^{2}$. Therefore, the actual values of $H^{(1)}$ and $H^{(2)}$ are 15.50 times those shown in Figs. 3 and 4.

It is our purpose to extend the present calculation to other levels of ${ }^{18} \mathrm{~F}$, and further, we expect to be able to apply the UPA to describe the $(d, n)$ stripping reaction on ${ }^{16} \mathrm{O}$. 
${ }^{1}$ E. O. Alt, W. Sandhas, and H. Ziegelmann, Phys. Rev. C 17, 1981 (1978).

${ }^{2}$ C. E. M. Aguiar, J. R. Brinati, and M. H. P. Martins, Nucl. Phys. A460, 381 (1986).

${ }^{3}$ G. H. Berthold, A. Stadler, and H. Zankel, Phys. Rev. C 41, 1365 (1990)

${ }^{4}$ P. Doleschall, H. Kröger, and R. J. Slobodrian, Phys. Rev. C 37, 927 (1988).

${ }^{5}$ D. J. Ernst, C. M. Shakin, and R. M. Thaler, Phys. Rev. C 8, 46 (1973).

${ }^{6}$ H. van Haeringen, J. Math. Phys. 24, 1274 (1983).
${ }^{7} \mathrm{H}$. van Haeringen, Charged-Particle Interactions (Coulomb, Leyden, 1985).

${ }^{8}$ B. A. Brown, S. E. Massen, and P. E. Hodgson, J. Phys. G 5, 1655 (1979).

${ }^{9}$ Y. Akiyama, Prog. Theor. Phys. 23, 903 (1960).

${ }^{10}$ K. Ueta, H. Miyake, and A. Mizukami, Phys. Rev. C 27, 389 (1983).

${ }^{11}$ H. Miyake, A. Mizukami, and K. Ueta, Nuovo Cimento A 84, 225 (1984).

${ }^{12}$ F. Ajzenberg-Selove, Nucl. Phys. A190, 1 (1972). 